\title{
Emitter Velocity Estimation Comparison for Frequency Difference of Arrival Measurement Based Single and Multiple Reference Lateration Algorithm
}

\author{
Abdulmalik Shehu Yaro ${ }^{1,2 *}$, Ahmad Zuri Sha'ameri ${ }^{1}$ \\ ${ }^{1}$ Faculty of Electrical Engineering, Universiti Teknologi Malaysia, 81310 UTM Skudai, Johor, Malaysia. \\ ${ }^{2}$ Department of Electrical and Computer Engineering, Ahmadu Bello University, Zaria, Main Campus, Nigeria.
}

"Corresponding author: yaroabdulmalik@ yahoo.com, Tel: +60103899873

\begin{abstract}
The accuracy at which the instantaneous velocity and position of a non-stationary emitting source estimated using a lateration algorithm depends on several factors such as the lateration algorithm approach, the number and choice of reference receiving station (RS) used in developing the lateration algorithm. In this paper, the use of multiple reference RSs was proposed to improve the velocity estimation accuracy of the frequency difference of arrival (FDOA) based lateration algorithm. The velocity estimation performance of the proposed multiple reference FDOA based lateration algorithm is compared with the conventional approach of using single reference RS at some selected emitter positions using Monte Carlo simulation. Simulation result based on an equilateral triangle RS configuration shows that the use of multiple reference RSs improved the velocity estimation accuracy of the lateration algorithm. Based on the selected emitter positions, a reduction in velocity estimation error of about $0.033 \mathrm{~m} / \mathrm{s}$ and $1.31 \mathrm{~m} / \mathrm{s}$ for emitter positions at ranges $0.5 \mathrm{~km}$ and $5 \mathrm{~km}$ respectively was achieved using the multiple reference lateration algorithm.
\end{abstract}

Keywords: FDOA; lateration algorithm; multiple reference; velocity estimation.

Article History: received 19 June 2017; accepted 21 March 2018; published 16 April 2018.

(C) 2018 Penerbit UTM Press. All rights reserved.

\section{INTRODUCTION}

Localization of non-stationary emitting source is an important topic with military and civil applications. It involves determining information about a non-stationary emitting source such as position and velocity from its electromagnetic emission detected at spatially placed receiving station $(\mathrm{RS}) \mathrm{s}$. It is a two-stage process $[1,2]$. In the first stage, emitter position dependent signal parameters such as time of arrival (TOA), frequency of arrival (FOA), received signal strength (RSS), time difference of arrival (TDOA), frequency difference of arrival (FDOA) and angle of arrival (AOA) measurements are estimated from the received electromagnetic emission. The next and final stage, which is the scope of this paper involves using the estimated position dependent signal parameter from the first stage with localization algorithm such as angulation, lateration and fingerprinting to determine the emitting source position and or velocity [1, 3]. The estimated position dependent signal parameter determined which information about the emitting source is to be determined as well as the localization algorithm to be used. The TOA, TDOA, RSS, and AOA measurements are used to determine the position of the emitting source while the FOA and FDOA measurements are used to determine the velocity of the emitting source $[4,5]$. The TOA, FOA,
TDOA and FDOA measurements are used with the lateration algorithm, RSS measurements are used with the fingerprinting while AOA measurements are used with the angulation algorithm $[2,6,7]$.

FDOA measurements have a non-linear relationship with the emitter velocity for this reason, several approaches have been developed to linearized this relationship [5, 8-19]. These approaches can be grouped in two, namely linear and non-linear approach $[2,20]$. The non-linear approach involves the use of linearization techniques to obtain the linear relationship between the input variable (FDOA measurement) and output variable (emitter velocity). This is followed by an iteration process subject to the minimization of a maximum likelihood cost function $[5,19]$. This approach to lateration algorithm is computationally complex, suffers from convergence issues due to the iteration process and is mostly implement in an active system [21]. The second approach which is adopted in this paper involves algebraic manipulation of the input and output variable to obtain a linear relationship $[8,10,13,15,18]$. This approach suffers no convergence issue as it does not involve iteration process and is mostly used in passive system, but is very sensitive to error in the input measurements [12, 22].

Several approaches have been proposed to improve velocity estimation of the lateration algorithm given 
perturb FDOA measurements [9-11]. The use of techniques such as approximate maximum likelihood (AML) algorithm [9], total least square (TLS) [11] and quadratic constraint solution (QCS) approaches [10] have been suggested. These techniques have shown great improvements in the velocity estimation accuracy of the lateration algorithm. The AML algorithm and QCS approach requires that the noise covariance matrix is known which is not available in passive systems and are mostly used with the non-linear approach FDOA based lateration algorithms. The TLS is used when both the dependent and independent variables are perturbed, but only the independent variables of the linear approach FDOA lateration algorithm are perturb. The use of multiple referencing approach to the lateration algorithm has also been suggested, but was only used for TDOA based lateration algorithm $[23,24]$. This paper proposed to improve the velocity estimation of the of the lateration algorithm using the multiple reference approach. The velocity estimation accuracy of the multiple reference FDOA based lateration algorithm was compared with the conventional approach of using single reference with three RSs using Monte Carlo simulation. It has been shown by Chan et al [25] that the best configuration for three RSs that will result in high estimation accuracy is an equilateral triangle. Thus, an equilateral triangular RS configuration with $2 \mathrm{~km}$ separation is considered for comparison.

The remainder of the paper is organized as follows: Section 2 gives a description of the velocity estimation methodology for the single and multiple reference FDOA based lateration algorithm. The simulation results and discussion are presented in Section 3 followed by the conclusion in Section 4.

\section{FDOA MEASUREMENT BASED LATERATION ALGORITHM}

This section of the paper presents the methodology for the single reference and multiple reference FDOA based lateration algorithms used to estimate the velocity of the non-stationary emitter. It is assumed that the location of the emitter is known, and all RSs are stationary.

\subsection{Single Reference FDOA based Lateration Algorithm}

Let the instantaneous location of the non-stationary emitter be $\mathbf{x}=(x, y)$ with an instantaneous velocity $\mathbf{v}=\left(u_{x}, u_{y}\right)$. The FOA at the $i$-th RS due its relative motion with the emitter is mathematically obtained as:

$f_{i}=\frac{f_{c}}{c} \times\left[\frac{\left(x-x_{i}\right) u_{x}}{R_{i}}+\frac{\left(y-y_{i}\right) u_{y}}{R_{i}}\right](\mathrm{Hz})$

for $i=1 \leq i \leq N$

where: $f_{c}$ is the carrier frequency of the signal in $\mathrm{Hz}, c=$ $3 \times 10^{8} \mathrm{~m} / \mathrm{s}, \mathbf{s}_{i}=\left(x_{i}, y_{i}\right)$ is the coordinate of the $i$-th RS and $R_{i}$ is the Euclidean instantaneous distance between the emitter and the $i$-th RS mathematically expressed as:

$R_{i}=\sqrt{\left(x-x_{i}\right)^{2}+\left(y-y_{i}\right)^{2}}$
Using the RS labelled 1 as reference and for $N=3$, the following FDOA equations are obtained.

$f_{12}=f_{1}-f_{2}$
$f_{13}=f_{1}-f_{3}$

Substituting equation (1) into equation (3), the FDOA equations in equation (3) are expressed as functions of the emitter position and instantaneous velocity as follows:

$$
\begin{aligned}
f_{12}= & \frac{f_{c}}{c} \times\left(\frac{\left(x-x_{1}\right) u_{x}}{R_{1}}+\frac{\left(y-y_{1}\right) u_{y}}{R_{1}}\right) \\
& -\frac{f_{c}}{c} \times\left(\frac{\left(x-x_{2}\right) u_{x}}{R_{2}}+\frac{\left(y-y_{2}\right) u_{y}}{R_{2}}\right) \\
f_{13} & =\frac{f_{c}}{c} \times\left(\frac{\left(x-x_{1}\right) u_{x}}{R_{1}}+\frac{\left(y-y_{1}\right) u_{y}}{R_{1}}\right) \\
& -\frac{f_{c}}{c} \times\left(\frac{\left(x-x_{3}\right) u_{x}}{R_{3}}+\frac{\left(y-y_{3}\right) u_{y}}{R_{3}}\right)
\end{aligned}
$$

From equation (4) and equation (5), the unknown variables are the instantaneous velocities $u_{x}$ and $u_{y}$. Further simplification of equation (4) and equation (5) results in equation (6) and equation (7) respectively.

$$
\begin{aligned}
& u_{x} \times a_{11}+u_{y} \times a_{12}=\frac{f_{12} \times c}{f_{c}} \\
& u_{x} \times a_{21}+u_{y} \times a_{22}=\frac{f_{13} \times c}{f_{c}}
\end{aligned}
$$

where:

$$
\begin{aligned}
& a_{11}=\frac{1}{R_{1}}\left(x-x_{1}\right)-\frac{1}{R_{2}}\left(x-x_{2}\right) \\
& a_{12}=\frac{1}{R_{1}}\left(y-y_{1}\right)-\frac{1}{R_{2}}\left(y-y_{2}\right) \\
& a_{21}=\frac{1}{R_{1}}\left(x-x_{1}\right)-\frac{1}{R_{3}}\left(x-x_{3}\right) \\
& a_{22}=\frac{1}{R_{1}}\left(y-y_{1}\right)-\frac{1}{R_{3}}\left(y-y_{3}\right)
\end{aligned}
$$

The FDOA equations presented in equation (6) and equation (7) when represented in matrix form is as follows:

$$
\mathbf{A}_{\text {single }} \mathbf{v}_{\text {single }}=\mathbf{b}_{\text {single }}
$$

where:

$$
\begin{aligned}
& \mathbf{A}_{\text {single }}=\left[\begin{array}{ll}
a_{11} & a_{12} \\
a_{21} & a_{22}
\end{array}\right] \\
& \mathbf{v}_{\text {single }}=\left[\begin{array}{l}
u_{x} \\
u_{y}
\end{array}\right]
\end{aligned}
$$




$$
\mathbf{b}_{\text {single }}=\left[\begin{array}{cc}
\frac{f_{12} \times c}{f_{c}} & \frac{f_{13} \times c}{f_{c}}
\end{array}\right]^{T}
$$

Using equation (9), the instantaneous velocity can be estimated using the FDOA measurements $f_{12}$ and $f_{13}$, and the coordinates of the RSs by finding the inverse matrix solution as follows:

$$
\mathbf{v}_{\text {single }}=\left(\mathbf{A}_{\text {single }}\right)^{-1} \mathbf{b}_{\text {single }}
$$

The solution to equation (11) is the estimated instantaneous velocity using the single reference FDOA based lateration algorithm.

\subsection{Multiple Reference FDOA based Lateration Algorithm}

In the multiple reference FDOA based lateration algorithm, a pair of RS is used as reference to obtain the FDOA measurements. Choosing the RS labelled 1 and 2 as reference pair, the FDOA equations obtained are:

$$
\begin{aligned}
& f_{13}=f_{1}-f_{3} \\
& f_{23}=f_{2}-f_{3}
\end{aligned}
$$

Comparing the FDOA equations in equation (3) and equation (12) presents a slight variation. From equation (12), it can be seen that $f_{23}$ replaced $f_{12}$ in equation (3). The FDOA equations for the multiple reference lateration algorithm in equation (12) as function of the emitter position and instantaneous velocity are:

$$
\begin{aligned}
f_{13}= & \frac{f_{c}}{c} \times\left(\frac{\left(x-x_{1}\right) u_{x}}{R_{1}}+\frac{\left(y-y_{1}\right) u_{y}}{R_{1}}\right) \\
& -\frac{f_{c}}{c} \times\left(\frac{\left(x-x_{3}\right) u_{x}}{R_{3}}+\frac{\left(y-y_{3}\right) u_{y}}{R_{3}}\right) \\
f_{23} & =\frac{f_{c}}{c} \times\left(\frac{\left(x-x_{2}\right) u_{x}}{R_{2}}+\frac{\left(y-y_{2}\right) u_{y}}{R_{2}}\right) \\
& -\frac{f_{c}}{c} \times\left(\frac{\left(x-x_{3}\right) u_{x}}{R_{3}}+\frac{\left(y-y_{3}\right) u_{y}}{R_{3}}\right)
\end{aligned}
$$

Further simplifications of equation (13) and equation (14) results in:

$$
\begin{aligned}
& u_{x} \times b_{11}+u_{y} \times b_{12}=\frac{f_{13} \times c}{f_{c}} \\
& u_{x} \times b_{21}+u_{y} \times b_{22}=\frac{f_{23} \times c}{f_{c}}
\end{aligned}
$$

where:

$$
b_{11}=\frac{1}{R_{1}}\left(x-x_{1}\right)-\frac{1}{R_{3}}\left(x-x_{3}\right)
$$

$$
\begin{aligned}
& b_{12}=\frac{1}{R_{1}}\left(y-y_{1}\right)-\frac{1}{R_{3}}\left(y-y_{3}\right) \\
& b_{21}=\frac{1}{R_{2}}\left(x-x_{2}\right)-\frac{1}{R_{3}}\left(x-x_{3}\right) \\
& b_{22}=\frac{1}{R_{2}}\left(y-y_{2}\right)-\frac{1}{R_{3}}\left(y-y_{3}\right)
\end{aligned}
$$

Rewriting equation (15) and equation (16) in matrix form as:

$$
\mathbf{A}_{\text {mult }} \mathbf{v}_{\text {mult }}=\mathbf{b}_{\text {mult }}
$$

where:

$$
\begin{aligned}
\mathbf{A}_{\text {mult }} & =\left[\begin{array}{ll}
b_{11} & b_{12} \\
b_{21} & b_{22}
\end{array}\right] \\
\mathbf{v}_{\text {mult }} & =\left[\begin{array}{l}
u_{x} \\
u_{y}
\end{array}\right] \\
\mathbf{b}_{\text {mult }} & =\left[\begin{array}{ll}
\frac{f_{13} \times c}{f_{c}} & \frac{f_{23} \times c}{f_{c}}
\end{array}\right]^{T}
\end{aligned}
$$

The instantaneous velocity for the multiple reference FDOA based lateration algorithm is obtained as:

$$
\mathbf{v}_{\text {mult }}=\left(\mathbf{A}_{\text {mult }}\right)^{-1} \mathbf{b}_{\text {mult }}
$$

In the next section, velocity estimation accuracy of the single reference and the multiple reference FDOA based lateration algorithm based on equation (11) and equation (20) are compared at some selected emitter positions.

\section{RESULTS AND DISCUSSION}

In this section, the accuracies in estimating the instantaneous velocity of a non-stationary emitter using the single reference FDOA based lateration algorithm in Section 2.1 and multiple reference FDOA based lateration algorithm in Section 2.2 are obtained and compared. Instantaneous velocity root mean square error (RMSE) is used as the performance measure to compare the performance of two lateration algorithms. Mathematically, the instantaneous velocity RMSE is obained follows:

$V_{\text {rmse }}=\sqrt{\frac{\sum_{i=1}^{N}\left[\left(\hat{u}_{x, i}-u_{x}\right)^{2}+\left(\hat{u}_{y, i}-u_{y}\right)^{2}\right]}{N}}$

where $\left(u_{x}, u_{y}\right)$ is the known instantaneous velocity and $\left(\hat{u}_{x, i}, \hat{u}_{y, i}\right)$ is the estimated instantaneous velocity at the $i$ th Monte Carlo iteration while $N$ is the total number of Monte Carlo simulation iterations. Monte Carlo simulation results are obtained after 500 iterations.

The FDOA error is modelled as a Gaussian distribution with zero mean and $\sigma$ standard deviation (SD). The estimated FDOA between the $i$-th and $j$-th $\mathrm{RS}$ pair is: 
$\hat{f}_{i j}=f_{i j}+N(0, \sigma)$

The distribution of the RSs used for the performance comparison is shown in Figure 1.

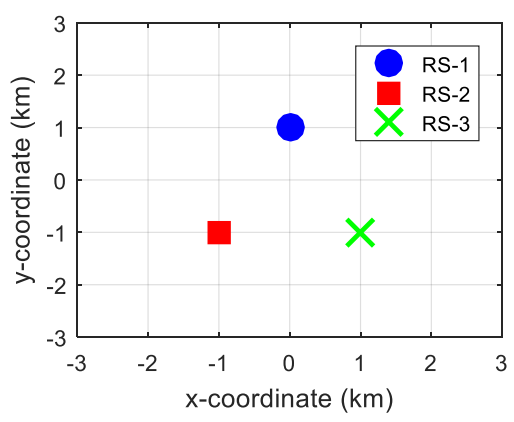

Figure 1. Two km equilateral triangle RS configuration

Navigation systems displace the locations of an emitting source in terms of range $(R)$ and bearing $(\theta)$ which corresponds to the cylindrical coordinate system. Conversion from cylindrical coordinate $\operatorname{system}(R, \theta)$ to the rectangular coordinate system $(x, y)$ is possible using equation (23).

$$
\begin{aligned}
& x=R \times \cos (\theta) \\
& y=R \times \sin (\theta)
\end{aligned}
$$

The instantaneous velocity estimation accuracy comparison was carried out at some selected emitter positions with coordinates and instantaneous velocity presented in Table 1.

Table 1. Selected emitter positions with their

\begin{tabular}{|c|c|c|c|c|}
\hline $\begin{array}{l}\text { Emitter } \\
\text { position }\end{array}$ & $\begin{array}{c}\text { Range } \\
(\mathrm{km})\end{array}$ & $\begin{array}{c}\text { Bearing } \\
\left({ }^{\circ}\right)\end{array}$ & $\begin{array}{l}\text { Velocity } \\
(\mathrm{km} / \mathrm{hr})\end{array}$ & $\begin{array}{c}\text { Velocity } \\
(m / s)\end{array}$ \\
\hline A & \multirow{4}{*}{0.5} & 0 & \multirow{2}{*}{$(36,36)$} & \multirow{2}{*}{$(10,10)$} \\
\hline B & & 30 & & \\
\hline $\bar{C}$ & & 60 & & \\
\hline $\mathrm{D}$ & & 90 & $(72,72)$ & $(20,20)$ \\
\hline $\mathrm{E}$ & \multirow{4}{*}{5} & 90 & \multirow{2}{*}{$(36,36)$} & \multirow{2}{*}{$(10,10)$} \\
\hline $\mathrm{F}$ & & 120 & & \\
\hline $\mathrm{G}$ & & 150 & \multirow{2}{*}{$(72,72)$} & \multirow{2}{*}{$(20,20)$} \\
\hline $\mathrm{H}$ & & 180 & & \\
\hline
\end{tabular}
instantaneous velocity.

Emitter at positions A, B, C, and D are located within the constellation of the RSs while E, F, G, and $\mathrm{H}$ are located outside the RS constellations. By varying the SD of the FDOA error from $0 \mathrm{~Hz}$ to $1 \mathrm{~Hz}$, the estimated instantaneous velocities obtained using the single reference FDOA based lateration algorithm are compared to that obtained using the multiple reference FDOA lateration algorithm at the selected emitter positions defined in Table 1. Figure 2 shows the velocity RMSE comparison between the two lateration algorithms. There is a linear relationship between the FDOA error SD and the velocity RMSE. The velocity RMSE increases with the
FDOA error $\mathrm{SD}$ from $0 \mathrm{~Hz}$ to $1 \mathrm{~Hz}$. Table 2 shows a summary of the velocity RMSE comparison between the two lateration algorithms at FDOA error SD of $0.5 \mathrm{~Hz}$.

Table 2. Velocity RSME comparison at FDOA error SD of $0.5 \mathrm{~Hz}$. Green shade indicates velocity estimation with the least estimation error.

\begin{tabular}{|c|c|c|c|}
\hline $\begin{array}{c}\text { Emitter } \\
\text { position }\end{array}$ & $\begin{array}{c}\text { Single } \\
\text { reference } \\
(\mathrm{m} / \mathrm{s})\end{array}$ & $\begin{array}{c}\text { Multiple } \\
\text { reference } \\
(\mathrm{m} / \mathrm{s})\end{array}$ & $\begin{array}{c}\text { Absolute } \\
\text { difference } \\
(\mathrm{m} / \mathrm{s})\end{array}$ \\
\hline $\mathrm{A}$ & 0.08 & 0.07 & 0.01 \\
\hline $\mathrm{B}$ & 0.09 & 0.08 & 0.01 \\
\hline $\mathrm{C}$ & 0.86 & 0.76 & 0.1 \\
\hline $\mathrm{D}$ & 0.08 & 0.07 & 0.01 \\
\hline $\mathrm{E}$ & 3.26 & 5.11 & 1.85 \\
\hline $\mathrm{F}$ & 6.59 & 5.51 & 1.08 \\
\hline $\mathrm{G}$ & 2.48 & 1.59 & 0.89 \\
\hline $\mathrm{H}$ & 5.61 & 3.65 & 1.96 \\
\hline
\end{tabular}

From Table 2, it can be seen that irrespective of the emitter bearing, increase in range increases the estimation error of both lateration algorithms. For instance, at emitter position A with an emitter range of $0.5 \mathrm{~km}$, the velocity RMSE for single and multiple reference lateration algorithms are $0.08 \mathrm{~m} / \mathrm{s}$ and $0.07 \mathrm{~m} / \mathrm{s}$ respectively, while at emitter position $F$ with $5 \mathrm{~km}$ range, the velocity RMSE for single and multiple reference lateration algorithms are $6.59 \mathrm{~m} / \mathrm{s}$ and $5.51 \mathrm{~m} / \mathrm{s}$. Velocity estimation accuracy comparison between the two lateration algorithms at emitter positions defined in Table 1 shows that the multiple reference lateration algorithm has the least estimation error except at emitter position E. At emitter position $\mathrm{C}$, the estimation error for the single and multiple reference lateration algorithms are $0.86 \mathrm{~m} / \mathrm{s}$ and $0.76 \mathrm{~m} / \mathrm{s}$ with an absolute error difference of $0.1 \mathrm{~m} / \mathrm{s}$. This means that a reduction of about $0.1 \mathrm{~m} / \mathrm{s}$ in estimating the velocity of the emitter can be achieved using the multiple reference lateration algorithm compared to the single reference lateration algorithm at that emitter position. On the average, based on the emitter positions considered, a decrease in error of about $0.033 \mathrm{~m} / \mathrm{s}$ was achieved at emitter positions with a range of $0.5 \mathrm{~km}$ irrespective of the bearing. As for the emitter positions with $5 \mathrm{~km}$ range, that is $\mathrm{F}, \mathrm{G}$ and $\mathrm{H}$, a decrease in error of about $1.31 \mathrm{~m} / \mathrm{s}$ was obtained with the multiple reference lateration algorithm. This means that on the average, the multiple reference approach improved the instantaneous velocity estimation of the lateration algorithm by a reduction in estimation error of about $0.033 \mathrm{~m} / \mathrm{s}$ and $1.31 \mathrm{~m} / \mathrm{s}$ for emitter at ranges $0.5 \mathrm{~km}$ and $5 \mathrm{~km}$ respectively based on the triangular RS configuration. 


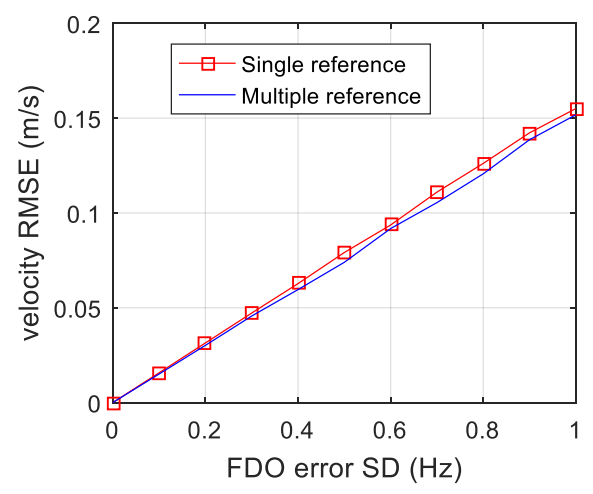

(a) Emitter position $\mathrm{A}$

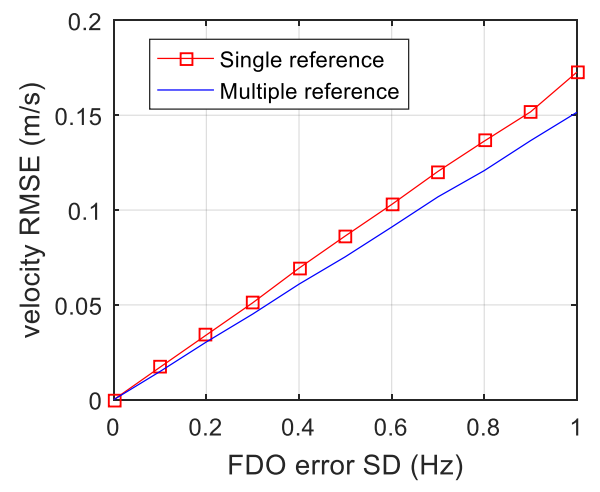

(c) Emitter position $\mathrm{C}$

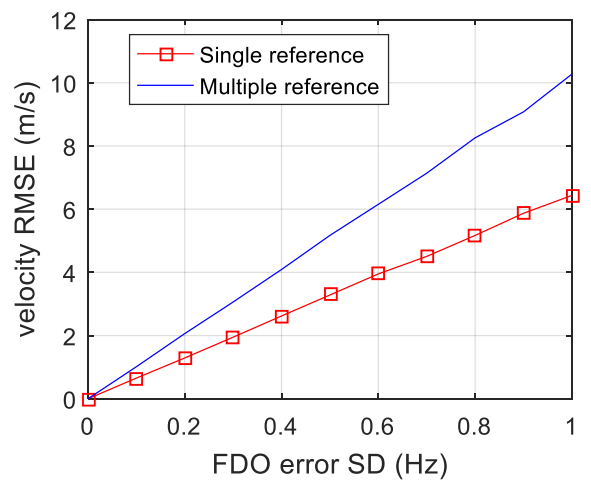

(e) Emitter position E

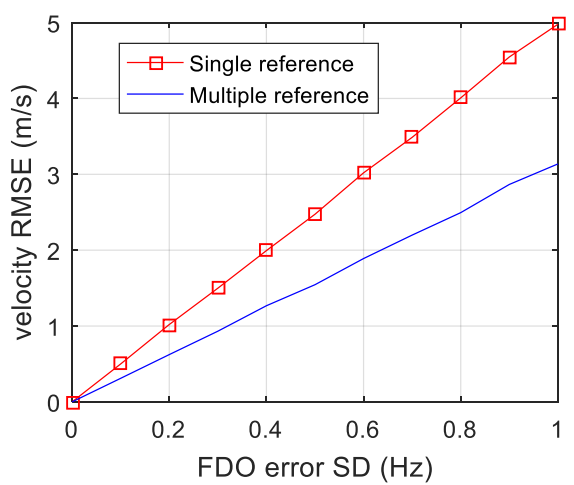

(g) Emitter position $\mathrm{G}$

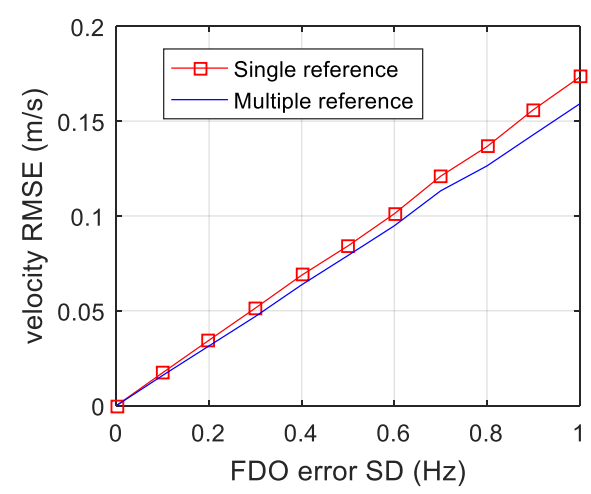

(b) Emitter position B

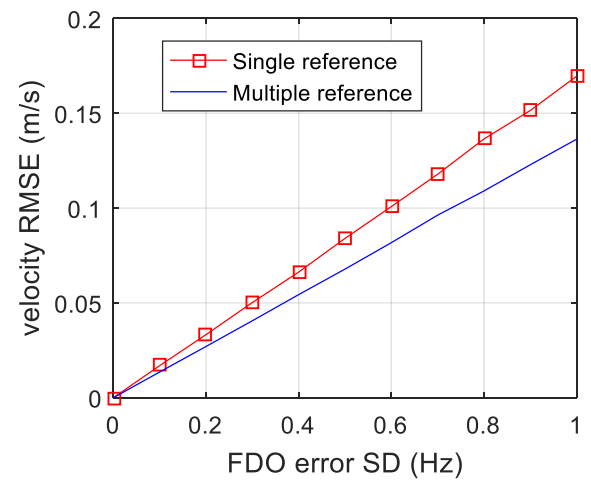

(d) Emitter position D

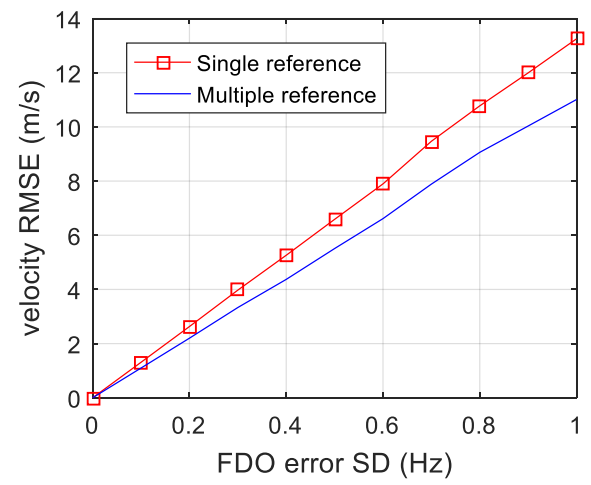

(f) Emitter position $\mathrm{F}$

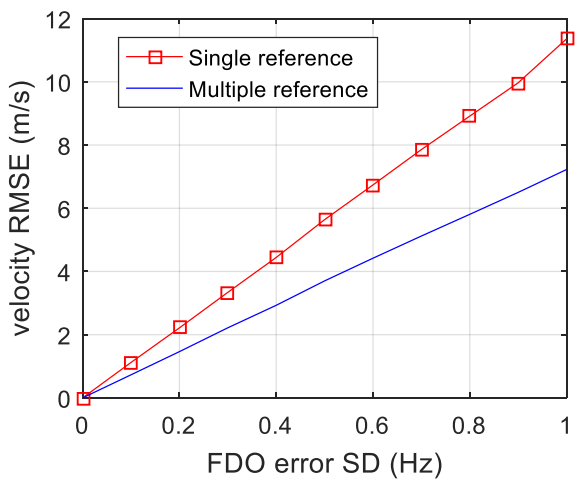

(h) Emitter position $\mathrm{H}$

Figure 2. Velocity estimation accuracy comparison for selected emitter positions 


\section{CONCLUSION}

In this paper, the lateration algorithm used in estimating the instantaneous velocity of an emitter using FDOA measurement based on single and multiple reference was developed. The accuracy in estimating the instantaneous velocity of the two lateration algorithms for some selected emitter position was determined with the RSs in a triangular configuration using Monte Carlo simulation for FDOA error SD range of $0 \mathrm{~Hz}$ to $1 \mathrm{~Hz}$. Simulation results show that for an emitter position with short range irrespective of the bearing, the instantaneous velocity estimation accuracy for both lateration algorithms was comparable. At large emitter range, the multiple reference lateration algorithm has the least estimation error compared to the single reference lateration algorithm. Thus, on the average, the multiple reference lateration algorithm approach has a better instantaneous velocity estimation accuracy compared to the single reference lateration algorithm for both short and long emitter ranges.

This paper considered only one set of reference, but it is believed that the choice of the reference to develop both lateration algorithms also influences the velocity estimation accuracy. Further work should be carried out to determine the effect of the choice of reference RS for both lateration algorithm and on a technique to choose the most suitable.

\section{REFERENCES}

[1] H. C. So, "Source localization: algorithms and analysis," in Handbook of Position Location: Theory, Practice, and Advances, John Wiley \& Sons, Inc., 2012, pp. 25-66.

[2] R. Zekavat and R. M. Buehrer, Handbook of position location: Theory, practice and advances. John Wiley \& Sons, Inc., 2012.

[3] W. H. L. Neven, T. J. Quilter, R. Weedo, and R. A. Hogendoorn, "Wide area multilateration (WAM)," Eurocontrol, Report on EATMP TRS 131/04 Version 1.1, 2005.

[4] J. Sadowski, "Velocity measurement using the FDOA method in ground-based radio navigation system," Metrology and Measurement Systems, vol. 21, no. 2, pp. 363-376, 2014.

[5] G.-H. Zhu, D.-Z. Feng, H. Xie, and Y. Zhou, "An approximately efficient bi-iterative method for source position and velocity estimation using TDOA and FDOA measurements," Signal Processing, vol. 125, pp. 110-121, Aug. 2016.

[6] A. S. Yaro and A. Z. Sha'meri, "Mathematical model of position estimation error for a Multiangulation System," in the 6th International Graduate Conference on Engineering, Science \& Humanity: Empowering innovation and Entrepreneurship for sustainable Development, 2016, pp. 134-136.

[7] A. Z. Sha'ameri, Y. A. Shehu, and W. Asuti, "Performance analysis of a minimum configuration multilateration system for airborne emitter position estimation," Defence S and T Technical Bulletin, vol. 8 , no. 1, pp. 27-41, 2015.

[8] K. C. Ho and W. Xu, "An accurate algebraic solution for moving source location using TDOA and FDOA measurements," IEEE Transactions on Signal Processing, vol. 52, no. 9, pp. 2453-2463, 2004.
[9] H. Yu, G. Huang, J. Gao, and X. Wu, “Approximate maximum likelihood algorithm for moving source lLocalization using TDOA and FDOA measurements," Chinese Journal of Aeronautics, vol. 25, no. 4, pp. 593-597, Aug. 2012.

[10] F. Quo and K. C. Ho, "A quadratic constraint solution method for TDOA and FDOA localization," in 2011 IEEE International Conference on Acoustics, Speech and Signal Processing (ICASSP), 2011, pp. 2588-2591.

[11] K. Yang, J. An, and Z. Xu, “A Quadratic Constraint Total Least-squares Algorithm for Hyperbolic Location," International Journal of Communications, Network and System Sciences, vol. 1, no. 2, pp. 130-135, 2008.

[12] G. Wang, S. Cai, Y. Li, and N. Ansari, "A biasreduced nonlinear WLS method for TDOA/FDOAbased source localization," IEEE Transactions on Vehicular Technology, vol. 65, no. 10, pp. 86038615, Oct. 2016.

[13] X. Sun, J. Li, P. Huang, and J. Pang, "Total leastsquares solution of active target localization using TDOA and FDOA measurements in WSN," in 22nd International Conference on Advanced Information Networking and Applications - Workshops (aina workshops 2008), 2008, pp. 995-999.

[14] J. Li, F. Guo, L. Yang, W. Jiang, and H. Pang, "On the use of calibration sensors in source localization using TDOA and FDOA measurements," Digital Signal Processing, vol. 27, no. 1, pp. 33-43, Apr. 2014.

[15] D.-G. Kim, Y.-H. Kim, and H.-N. Kim, “Two-step estimator for moving-emitter geolocation using time difference of arrival/frequency-difference of arrival measurements," IET Radar, Sonar \& Navigation, vol. 9, no. 7, pp. 881-887, 2015.

[16] H. Yu, G. Huang, J. Gao, and B. Yan, "Practical constrained least-square algorithm for moving source location using TDOA and FDOA measurements," Journal of Systems Engineering and Electronics, vol. 23, no. 4, pp. 488-494, Aug. 2012.

[17] X. Qu and L. Xie, "An efficient convex constrained weighted least squares source localization algorithm based on TDOA measurements," Signal Processing, vol. 119, pp. 142-152, Feb. 2016.

[18] K. C. Ho, X. Lu, and L. Kovavisaruch, "Source localization using TDOA and FDOA measurements in the presence of receiver location errors: Analysis and solution," IEEE Transactions on Signal Processing, vol. 55, no. 2, pp. 684-696, 2007.

[19] H. Yu, G. Huang, J. Gao, and B. Liu, "An efficient constrained weighted least squares algorithm for moving source location using TDOA and FDOA measurements," IEEE Transactions on Wireless Communications, vol. 11, no. 1, pp. 44-47, Jan. 2012.

[20] A. S. Yaro, A. Z. Sha'ameri, and N. Kamel, "Ground receiving station reference pair selection technique for a minimum configuration $3 \mathrm{D}$ emitter position estimation multilateration system," Advances in Electrical and Electronic Engineering, vol. 15, no. 3, pp. 391-399, Oct. 2017.

[21] D. E. Chaitanya, M. N. V. S. S. Kumar, G. S. Rao, and R. Goswami, "Convergence issues of taylor 
series method in determining unknown target location using hyperbolic multilateration," 2014 International Conference on Science Engineering and Management Research, ICSEMR 2014, no. 1, pp. 1-4, 2015.

[22] K. C. Ho, "Bias reduction for an explicit solution of source localization using TDOA," IEEE Transactions on Signal Processing, vol. 60, no. 5, pp. 2101-2114, 2012.

[23] H. Torbati Fard, M. Atashbar, Y. Norouzi, and F. Hojjat Kaskani, "Multireference TDOA-based source localization," Turkish Journal of Electrical Engineering \& Computer Sciences, vol. 21, pp. 1920-1929, 2013.

[24] M. D. Gillette and H. F. Silverman, "A linear closedform algorithm for source localization from timedifferences of arrival," IEEE Signal Processing Letters, vol. 15, no. 1, pp. 1-4, 2008.

[25] Y. Chen, J. A. Francisco, W. Trappe, and R. P. Martin, "A practical approach to landmark deployment for indoor localization," in 2006 3rd Annual IEEE Communications Society on Sensor and Ad Hoc Communications and Networks, 2006, vol. 1, pp. 365-373. 